\title{
SIMULATION OF JET QUENCHING IN HEAVY ION COLLISIONS
}

\author{
I. P. LOKHTIN* and A. M. SNIGIREV \\ Skobeltsyn Institute of Nuclear Physics, Moscow State University, Moscow, 119992, Russia \\ *E-mail: Igor.Lokhtin@cern.ch
}

\begin{abstract}
The method to simulate the rescattering and energy loss of hard partons in heavy ion collisions has been developed. The model is a fast Monte-Carlo tool introduced to modify a standard PYTHIA jet event. The full heavy ion event is obtained as a superposition of a soft hydro-type state and hard multi-jets. The model is applied to analysis of the jet quenching pattern at RHIC and LHC.
\end{abstract}

Keywords: quark-gluon plasma; partonic energy loss; jet quenching.

\section{Introduction}

One of the important tools for studying the properties of quark-gluon plasma (QGP) in ultrarelativistic heavy ion collisions is the analysis of a QCD jet production. The medium-induced energy loss of energetic partons, "jet quenching", should be very different in the cold nuclear matter and QGP, resulting in many observable phenomena ${ }^{1}$. Recent RHIC data on high-p p $_{T}$ particle production at $\sqrt{s}=200 \mathrm{~A} \mathrm{GeV}$ are in agreement with the jet quenching hypothesis (see, e.g., ${ }^{2}$ and references therein). At LHC, a new regime of heavy ion physics will be reached at $\sqrt{s_{\mathrm{NN}}}=5.5 \mathrm{~A} \mathrm{TeV}$ where hard and semi-hard particle production can stand out against the underlying soft events. The initial gluon densities in $\mathrm{Pb}+\mathrm{Pb}$ reactions at LHC are expected to be much higher than those at RHIC, implying a stronger partonic energy loss, observable in new channels.

In the most of available Monte-Carlo heavy ion event generators the mediuminduced partonic rescattering and energy loss are either ignored or implemented insufficiently. Thus, in order to analyze RHIC data on high- $\mathrm{p}_{T}$ hadron production and test the sensitivity of LHC observables to the QGP formation, the development of adequate and fast Monte-Carlo tool to simulate the jet quenching is necessary.

\section{Physics model and simulation procedure}

The detailed description of physics model can be found in our recent paper ${ }^{3}$. The approach bases on an accumulating energy loss, the gluon radiation being associated with each parton scattering in the expanding medium and includes the interference effect using the modified radiation spectrum $d E / d l$ as a function of decreasing temperature $T$. The basic kinetic integral equation for the energy loss $\Delta E$ as a function of initial energy $E$ and path length $L$ has the form

$$
\Delta E(L, E)=\int_{0}^{L} \frac{d l}{\lambda} \frac{d P}{d l} \frac{d E}{d l}, \frac{d P}{d l}=\frac{\mathrm{e}^{-l / \lambda}}{\lambda}
$$

where $l$ is the current transverse coordinate of a parton, $d P / d l$ is the scattering probability density, $d E / d l$ is the energy loss per unit length, $\lambda$ is in-medium mean free path. The collisional loss in high-momentum transfer limit and radiative loss in BDMS approximation ${ }^{4}$ (with "dead-cone" generalization of the radiation spectrum for heavy quarks ${ }^{5}$ ) are using. We consider realistic nuclear geometry ${ }^{6}$ and treat the medium in nuclear overlapping zone as a boost-invariant longitudinally expanding quark-gluon fluid. The model parameters are the initial conditions for the QGP formation for central $\mathrm{Au}+\mathrm{Au}(\mathrm{Pb}+\mathrm{Pb})$ collisions at RHIC (LHC): 
the proper formation time $\tau_{0}$ and the temperature $T_{0}$. For non-central collisions we suggest the proportionality of the initial energy density $\varepsilon_{0}$ to the ratio of nuclear overlap function and transverse area of nuclear overlapping ${ }^{6}$. The simple Gaussian parameterization of gluon angular distribution over the emission angle $\theta$ with the typical angle of the coherent radiation $\theta_{0} \sim 5^{0}$.

The model has been constructed as the Monte-Carlo event generator PYQUEN (PYthia QUENched) and is available via Internet $^{8}$. The routine is implemented as a modification of the standard PYTHIA_6.4 jet event ${ }^{9}$. The event-by-event simulation procedure includes the generation of the initial parton spectra with PYTHIA and production vertexes at given impact parameter, rescattering-by-rescattering simulation of the parton path length in a dense zone, radiative and collisional energy loss per rescattering, final hadronization with the Lund string model for hard partons and in-medium emitted gluons.

The full heavy ion event is simulated as a superposition of soft hydro-type state and hard multi-jets. The simple approximation ${ }^{3}$ of hadronic liquid at "freeze-out" stage has been used to treat soft part of the event. Then the hard part of the event includes PYQUEN multi-jets generated according to the binomial distribution. The mean number of jets produced in AA events at a given impact parameter is a product of the number of binary NN sub-collisions and the integral cross section of hard process in $p p$ collisions with the minimal transverse momentum transfer $p_{T}^{\min }$. The extended in such a way model has been also constructed as the fast Monte-Carlo event generator ${ }^{10}$. Note that ideologically similar approximation has been developed in ${ }^{11}$.

\section{Jet quenching at RHIC}

In order to demonstrate the efficiency of the model, the jet quenching pattern in $\mathrm{Au}+\mathrm{Au}$ collisions at RHIC was considered. The PHOBOS data on $\eta$-spectra of charged hadrons ${ }^{12}$ have been analyzed at first to fix the particle density in the mid-rapidity and the maximum longitudinal flow rapidity, $Y_{L}^{\max }=3.5$ (figure 1). To calculate multijet production cross section, the higher order corrections factor $K=2$ was used. The

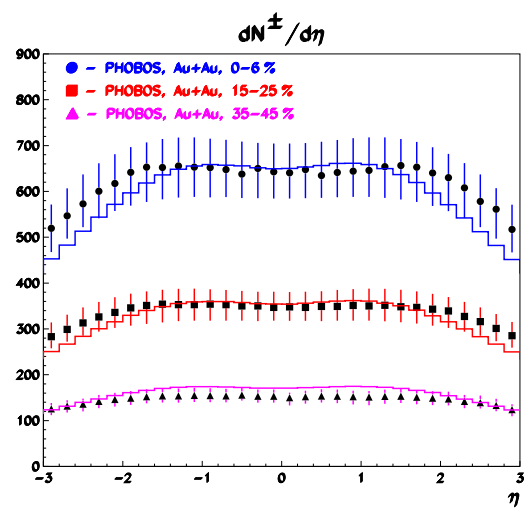

Fig. 1. The pseudorapidity distribution of charged hadrons in $\mathrm{Au}+\mathrm{Au}$ collisions for three centrality sets. The points are PHOBOS data, histograms are the model calculations.

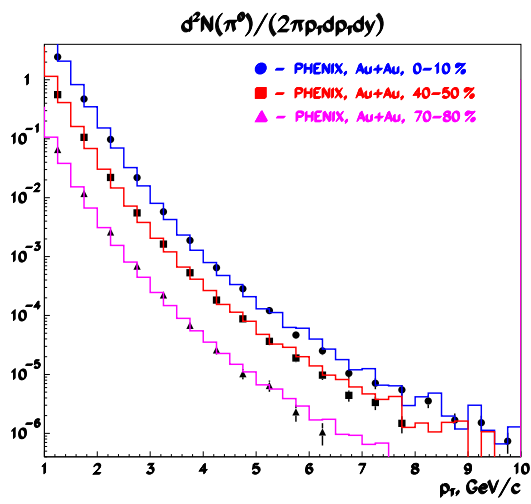

Fig. 2. The transverse momentum distribution of neutral pions in $\mathrm{Au}+\mathrm{Au}$ collisions for three centrality sets. The points are PHENIX data, histograms are the model calculations. 
rest of the model parameters have been obtained by fitting PHENIX data on $p_{T}$-spectra of neutral pions ${ }^{13}$ (figure 2): the kinetic freeze-out temperature $T_{f}=100 \mathrm{MeV}$, maximum transverse flow rapidity $Y_{T}^{\max }=1.25$ and $p_{T}^{\min }=2.8 \mathrm{GeV} / c$. The nuclear modification of the hardest domain of $p_{T}$-spectrum was used to extract initial QGP conditions: $T_{0}=500 \mathrm{MeV}$ and $\tau_{0}=0.4 \mathrm{fm} / c$. Figure 3 shows that our model reproduce $p_{T^{-}}$and cen-

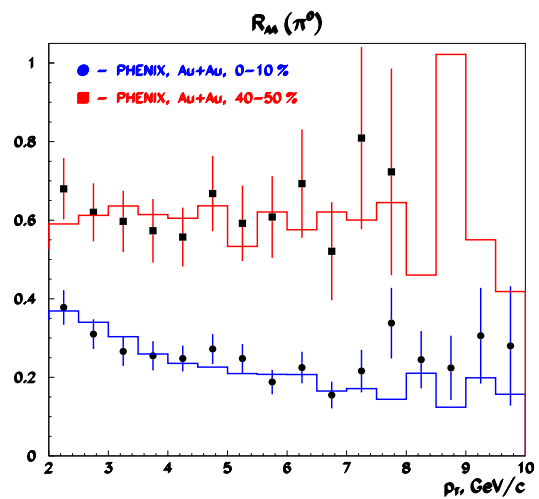

Fig. 3. The nuclear modification factor $R_{A A}$ for neutral pions in $\mathrm{Au}+\mathrm{Au}$ collisions for two centrality sets. The points are PHENIX data, histograms are the model calculations.

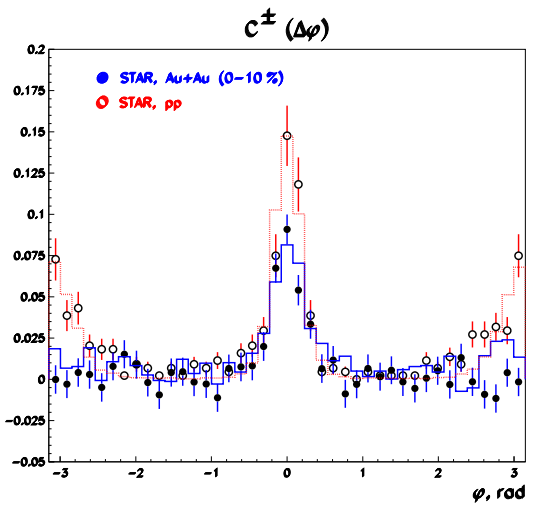

Fig. 4. The azimuthal two-particle correlation function for $p p$ and for central $\mathrm{Au}+\mathrm{Au}$ collisions. The points are STAR data, dashed and solid histograms are the model calculations for $p p$ and $\mathrm{Au}+\mathrm{Au}$ events respectively. trality dependences of nuclear modification factor $R_{A A}$ (determined as a ratio of particle yields in $A A$ and $p p$ collisions normalized on the number of binary NN sub-collisions) quite well.

Another important tool to verify jet quenching is two-particle azimuthal correlation function $C(\Delta \varphi)$ - the distribution over an azimuthal angle of high- $p_{T}$ hadrons in the event with $2 \mathrm{GeV} / c<p_{T}<p_{T}^{\text {trig }}$ relative to that for the hardest "trigger" particle with $p_{T}^{\text {trig }}>4 \mathrm{GeV} / c$. Figure 4 presents $C(\Delta \varphi)$ in $p p$ and in central $\mathrm{Au}+\mathrm{Au}$ collisions (data from STAR ${ }^{14}$ ). Clear peaks in $p p$ collisions at $\Delta \varphi=0$ and $\Delta \varphi=\pi$ indicate a typical dijet event topology. However, for central $\mathrm{Au}+\mathrm{Au}$ collisions the peak near $\pi$ disappears. It can be interpreted as the observation of monojet events due to the absorption of one of the jets in a dense medium. Figure 4 demonstrates that measured suppression of azimuthal back-to-back correlations is well reproduced by our model.

We leave beyond the scope of this paper the analysis of such important RHIC observables as the azimuthal anisotropy and particle ratios, which are sensitive to the soft physics. In order to study them, a more careful treatment of low- $p_{T}$ particle production than our simple approach is needed (the detailed description of space-time structure of freeze-out region, resonance decays, etc.).

\section{Jet quenching at LHC}

The developed model was applied to analyze various novel features of jet quenching at the LHC (see ${ }^{15,16,17}$ for details). Let us just to enumerate some main issues.

Jets tagged by leading hadrons. The relation between in-medium softening jet fragmentation function (measured in leading hadron channel) and suppression of jet rates due to energy loss out of jet cone, was analyzed in our work ${ }^{15}$. We have found that the specific anti-correlation between two 
effects allows one to probe parton energy loss mechanism (small-angular radiation vs. wide-angular radiation and collisional loss).

Jets induced by heavy quarks. The possibility to observe the medium-modified fragmentation of hard b-quarks tagged by a energetic muon in heavy ion collisions was analyzed in the work ${ }^{16}$. We have found that the reasonable statistics, $\sim 10^{4}$ events per one month of LHC run with lead beams, can be expected for the realistic geometrical acceptance and kinematic cuts. The significant softening b-jet fragmentation function determined by the absolute value of partonic energy loss and the angular radiation spectrum is predicted.

$Z / \gamma^{*}+$ jet production. The channel with dimuon tagged jet production in heavy ion collisions was analyzed in the work ${ }^{17}$. The correlations between $\mu^{+} \mu^{-}$pair and jet, as well as between $\mu^{+} \mu^{-}$pair and a leading particle in a jet, were first numerically studied. We have found that the medium-induced partonic energy loss can result in significant $P_{T}$-imbalance between $\mu^{+} \mu^{-}$pair and a leading particle in a jet, which is quite visible even for moderate loss.

\section{Conclusions}

The method to simulate jet quenching in heavy ion collisions has been developed. The model is the fast Monte-Carlo tool implemented to modify a standard PYTHIA jet event. The full heavy ion event is obtained as a superposition of a soft hydro-type state and hard multi-jets. The model is capable of reproducing main features of the jet quenching pattern at RHIC (the $p_{T}$ dependence of the nuclear modification factor and the suppression of azimuthal back-to-back correlations). The model was also applied to probe jet quenching in various new channels at LHC energy: jets tagged by leading particles, b-jets, dilepton-jet correlations. The further development of the model focusing on a more detailed description of low- $p_{T}$ particle production is planned for the future.

\section{Acknowledgments}

Discussions with A. Morsch, C. Roland, L.I. Sarycheva, J. Schukraft and B. Wyslouch are gratefully acknowledged. This work is supported by grant N 04-02-16333 of Russian Foundation for Basic Research and Contract N 02.434.11.7074 of Russian Ministry of Science and Education.

\section{References}

1. R. Baier, D. Schiff and B.G. Zakharov, Annual Rev. Nucl. Part. Sci. 50, 37 (2000).

2. X.-N. Wang, Phys. Lett. B579, 299 (2004).

3. I.P. Lokhtin and A.M. Snigirev, Eur. Phys. J. C45, 211 (2006).

4. R. Baier, Yu. L. Dokshitzer, A.H. Mueller and D. Schiff, Phys. Rev. C60, 064902 (1999).

5. Yu.L. Dokshitzer and D. Kharzeev, Phys. Lett. B519, 199 (2001).

6. I.P. Lokhtin and A.M. Snigirev, Eur. Phys. J. C 16, 527 (2000).

7. I.P. Lokhtin and A.M. Snigirev, Phys. Lett. B 440, 163 (1998).

8. http://cern.ch/lokhtin/pyquen .

9. T. Sjostrand, Comp. Phys. Com. 135, 238 (2001)

10. http://cern.ch/lokhtin/hydro/hydjet.html .

11. T. Hirano and T. Nara, Nucl. Phys. A743, 305 (2004).

12. B.B. Back et al. (PHOBOS Collab.), Phys. Rev. Lett. 91, 052303 (2003).

13. S.S. Adler et al. (PHENIX Collab.), Phys. Rev. Lett. 91, 072301 (2003).

14. C. Adler et al. (STAR Collaboration), Phys. Rev. Lett. 89, 202301 (2002).

15. I.P. Lokhtin and A.M. Snigirev, Phys. Lett. B567, 39 (2003).

16. I.P. Lokhtin, L.I. Sarycheva, A.M. Snigirev and K.Yu. Teplov, Eur. Phys. J. C37, 465 (2004).

17. I.P. Lokhtin, A.V. Sherstnev and A.M. Snigirev, Phys. Lett. B599, 260 (2004). 